M. Farooqui: None. S. Dandapat: None. S. Ortega Gutierrez: 2; C; Medtronic, Stryker.

\section{E-092 EFFECT OF STENT RETRIEVER THROMBECTOMY VS. COMBINED TECHNIQUE ON REVASCULARIZATION IN PATIENTS WITH LARGE VESSEL OCCLUSION STROKE: CASE-CONTROL STUDY}

M Mohammaden*, D Haussen, L Pisani, A Al-Bayati, A Anderson, B Liberato, M Frankel, R Nogueira. Marcus Stroke and Neuroscience Center, Grady Memorial Hospital and Department of Neurology, Emory University School of Medicine, Atlanta, GA

\subsection{6/neurintsurg-2020-SNIS. 125}

Background and Purpose Stent-retriever (SR) thrombectomy remains the best proven treatment modality for anterior circulation large vessel occlusion strokes (LVOS). Three randomized clinical trials have reported similar safety and efficacy for contact aspiration (CA) and SR thrombectomy. The ASTER-2 trial compared combined technique (SR + CA) versus SR alone. The study failed to demonstrate differences in terms of final reperfusion and clinical outcomes across the two modalities. However, reperfusion was significantly better after the allocated treatment only with combined technique. We aim to determine whether the combined technique is superior to SR thrombectomy alone as first line strategy in a large single-center cohort where balloon-guide catheter was universally used.

Methods A prospectively maintained mechanical thrombectomy database from January 2018 to December 2019 was reviewed. Patients were included if they had anterior circulation LVO due to intracranial ICA or MCA-M1 segment and underwent SR thrombectomy or combined technique as first-line therapy. The cohort was divided into two groups matched for age, baseline NIHSS score, prior IV-tPA, site of occlusion, stroke etiology, use of general anesthesia and balloon guide catheter. The primary outcome was the achievement of first-pass effect (FPE) (mTICI2c-3). Secondary outcomes included modified FPE, number of passes, successful reperfusion (mTICI2b-3) without and after rescue techniques and functional independence (90-day mRS 0-2). Safety outcomes included the rates of parenchymal hematoma (PH) type 2 and 90-day mortality. A secondary analysis was performed for the interaction between different subgroups and thrombectomy modality on successful reperfusion.
Results A total of 244 patients (122 pairs) were eligible for the analysis. Patients with first-line combined technique had similar rates of FPE $(56.6 \%$ vs. $51.6, \mathrm{p}=0.44)$, successful reperfusion without $(77 \%$ vs. $77.9 \%, \quad \mathrm{p}=0.88)$ and after (99.2\% vs. $99.2 \%, p=1.00)$ rescue techniques and 90 -day functional independence $(46.6 \%$ vs. $54.4 \%, \mathrm{p}=0.30)$ compared to SR thrombectomy. However, first-line combined technique had a significantly higher number of passes and longer reperfusion times compared to SR alone $(\mathrm{p}=0.005$ and $\mathrm{p}=0.01$, respectively). The rates of $\mathrm{PH}$ type 2 and 90-day mortality were comparable between both groups. There was no significant interaction between age, prior IV-tPA, site of occlusion and presence of favorable collaterals and the first-line treatment modality in terms of successful reperfusion.

Conclusions Our findings reinforce the findings of ASTER-2 in that the first-line thrombectomy with a combined technique did not result in increased rates of successful reperfusion or a better outcome. In contrast with ASTER-2, we did not see any advantage even prior to rescue devices.

Disclosures M. Mohammaden: None. D. Haussen: 2; C; Stryker, Vesalio. 4; C; Viz.AI. L. Pisani: None. A. Al-Bayati: None. A. Anderson: None. B. Liberato: None. M. Frankel: None. R. Nogueira: 2; C; Medtronic, Anaconda, Genentech, Brainomix, Viz-AI, Corindus Vascular Robotics.

\section{E-093 ENDOVASCULAR TREATMENT OF ACUTE CAROTID ATHEROSCLEROTIC TANDEM OCCLUSIONS: PREDICTORS OF CLINICAL OUTCOMES AS TECHNICAL ASPECTS AND LOCATION OF TANDEM OCCLUSIONS}

${ }^{1} \mathrm{E}$ Yoon, ${ }^{2} \mathrm{~S} \mathrm{Ha}^{*} .{ }^{1}$ Radiology, Chosun univ. Hospital, Gwangju Metropolitan City, Korea, Republic of; ${ }^{2}$ Neurosurgery, Chosun univ. Hospital, Gwangju Metropolitan City, Korea, Republic of

\subsection{6/neurintsurg-2020-SNIS. 126}

Objective We aimed to analyze angiographic and clinical outcomes according to the sequence of treatment (antegrade versus retrograde) in patients with acute ischemic stroke caused by tandem extracranial cervical carotid and intracranial large vessel occlusion.

Methods All eligible tandem occlusion patients from April 2012 to March 2019 undergoing carotid artery stenting (CAS) simultaneously with intracranial endovascular thrombectomy (EVT) were retrospectively reviewed. After dividing into 2 